\title{
Self-organized supply chain networks: Theory in practice and an analog simulation based approach
}

\author{
K. R. Anne \\ Institute for Smart Systems- \\ Technologies \\ University of Klagenfurt \\ 9020, Klagenfurt, Austria
}

rao.anne@uni-klu.ac.at

\author{
S. K. Bhagavatula \\ Institute for Smart Systems- \\ Technologies \\ University of Klagenfurt \\ 9020, Klagenfurt, Austria
}

sreeram.kumar@uni-klu.ac.at

\author{
K. Kyamakya \\ Institute for Smart Systems- \\ Technologies \\ University of Klagenfurt \\ 9020, Klagenfurt, Austria \\ kyandoghere.kyamakya@uni-

\section{lu.ac.at}

\begin{abstract}
Global supply chain networks are undergoing a transition with mass customization policies, shrinking profit margins, non deterministic order behavior together with other uncertainties. Self-organized supply chain networks (SCN) are offering an alternative as they enjoy the flexibility needed to respond in real time. In this paper, we describe the pre-requisites for the selforganization of a SCN. This paper also proposes a nonlinear model for the SCN. A brief state of the art towards the selforganized supply chains is presented illustrating the theory that is in practice. It is shown that synchronization is a vital step towards the self-organization and different aspects of synchronization are discussed. The major contribution is towards the analog simulation of the supply chain model in consideration using the cellular neural networks (CNN). The performance comparison with the numerical simulation is also discussed. Today companies expect to use the modern information and communication technologies to achieve the efficient supply chain networks. In this work we are dealing with the depth and reliability of information available with these technologies coupled with the individual objectives of the companies and proposing an analog simulation based approach to provide solution in real time. Our findings concern future supply chain management practices, a new research directions.
\end{abstract}

\section{Categories and Subject Descriptors}

B.2.2 [Arithmetic and logic structures]: Performance Analysis and Design Aids - Simulation, Verification.

\section{General Terms}

Management, Design, Economics.

\section{Keywords}

Self-organized supply chains, Adaptive supply chains, Supply chain optimization, and Analog simulation

Permission to make digital or hard copies of all or part of this work for personal or classroom use is granted without fee provided that copies are not made or distributed for profit or commercial advantage and that copies bear this notice and the full citation on the first page. To copy otherwise, to republish, to post on servers or to redistribute to lists, requires prior specific permission and/or a fee. AUTONOMICS 2008, September 23-25, Turin, Italy

Copyright (C) 2008 ICST 978-963-9799-34-9

DOI 10.4108/ICST.AUTONOMICS2008.4672

\author{
J. C. Chedjou \\ Institute for Smart Systems- \\ Technologies \\ University of Klagenfurt \\ 9020, Klagenfurt, Austria \\ jean.chedjou@uni-klu.ac.at
}

\section{INTRODUCTION}

The traditional supply chain management has been based on limited information sharing restricted to the product in consideration and transaction oriented towards that product [1]. The relation between the entities is often characterized by the mistrust and competition. The modern supply chains are global in nature and coupled with each other, however the degree of coupling varies from entity to entity $[2,3]$. The traditional "arm's - length" relationship between entities should be changed to "durable arm's - length" relationships [4]. The objective of any entity in the supply chain network is to achieve maximum profits and give maximum customer satisfaction. In addition to the stated individual objective all entities also have a responsibility towards the global objective of resilient supply chain network (SCN). This realization has made the entities in SCN to look beyond their own boundaries to asses how the resources of each other can be utilized to achieve the global objective with out compromising on their own objective. In the midst of pursing towards it, entities have relentlessly restructured and reengineered their internal organizational boundaries and policies. However, during this processes companies with in the SCN realized that they need to achieve the self organization of the SCN they belong in order to satisfy the stated objective.

In accordance with the greater focus on self-organization of the supply chain, the companies are increasingly focusing on the prerequisitions like, integration, collaboration and synchronization between all entities in the SCN.

The first step towards self-organized supply chains is integrated stage. Intra-entity integration also called as functional integration is the basic driver towards the integration of the entire SCN. Functional integration of purchasing, manufacturing, transportation, and warehousing activities gives the very much needed visibility to the SCN. Besides functional integration intertemporal coupling, also called hierarchical integration, of these activities over strategic, tactical, and operational levels is also important [1, 2, 5]. This inter-temporal coupling requires consistency among overlapping supply chain decisions at various levels of planning. However the major role of the inter-temporal 
integration is in product life cycle design. Improved integration of activities across multiple companies/entities in a supply chain is a concern of increasing importance [6]. Information technology is the key enabler for the integration in supply chain. The information systems provide the necessary information needed for the integration.

After the integration of the supply chain, the next step towards self-organization is collaboration between entities. Of course collaboration concepts are not new, they exists from the days of traditional business in the form of contracts. However the effectiveness, execution speed, of the collaborations is highly increased due the technological advances and the integration tools. As more and more supply chains need to cater towards the mass customization, make-to-order, build-to-order and configureto-order, the effective collaboration between entities is also a priority. Depending upon the model in use the processes to include in the collaboration varies. Degree of collaboration varies depending upon the processes included and the strength of the integration [7]. Collaboration is strong where business to business relation ships are strong.

The true collaboration among and in between all the entities in a SCN is more difficult in practice. There are various external and internal perturbations to the information flow with in the supply chain. These perturbations can force the systems into chaotic state or saturation state $[2,8]$. Synchronization of the SCN is a vital step towards the self organization. Uncertainties and exceptions are identified early and the data for intelligent response is immediately available. This greatly minimizes the bullwhip effect, demand amplification, and saves downstream partners and customers from needless activity [2, 3, 8, 9]. The synchronization can be classified into two types: namely complete synchronization and partial synchronization. Complete synchronization enables the supply chain to react quickly to changes in demand and in product design. This type of synchronization is particularly suitable in just-in-time supply chain networks. To achieve the complete synchronization the complete chain should follow the integration and collaboration methods in true spirit. Partial synchronization is achieved through the controller item. Apart from the data synchronization as explained in complete synchronization, a controller item is developed to mitigate the effect caused due to time lag. In this synchronization major effort is placed in modeling the effect and quantifying it. The modeling and quantification of the effect caused by the time lag, information discrepancy, and individual objectives helps in designing the controller/synchronizer element. This controller item can be unique for each entity or supply chain as a whole.

The structure of the paper is as follows. In section 2, we discuss various SCN models in general and in particular the complex nonlinear model we are using in this work. The key enablers for the self-organized supply chains together with the state of the art towards the self-organized SCN are briefly discussed in section 3 . The importance of analog simulation based on cellular neural networks $(\mathrm{CNN})$ in achieving the self-organized SCN is discussed in section 4. Section 4 also includes the results obtained with CNN. Section 5 deals with the conclusions and proposals for future work.

\section{SCN modeling}

The theoretical framework for the supply chain management underlies the setting, optimization and control of the system model. The system model is not unique for all the SCNs [10]. The system dynamics change for each type, food, oil, consumer goods, etc., of SCN depending upon the processes involved. System dynamics based approach to model the business dynamics was first introduced by Forrester [11]. System Dynamics has its origins in control engineering and management; the approach uses a perspective based on information feedback and delays to understand the dynamic behavior of complex physical and social systems. System dynamics are actively used to model the managerial behavior. In 1989, Sterman [12] proposed a model that can be used to model the supply chains using the industrial dynamics fundamentals proposed by Forrester. This [12], Sterman model was actively used to model the supply chain dynamics. Due to its visual nature and simplicity, after a lengthy floppy period, system dynamic approach is gaining momentum in modeling the inventory management process, policy development and demand amplification [13]. System theory based modeling is also used to develop the feedback controllers to mitigate the bullwhip effect, demand amplification, to some extent [14]. However to address the issues emanating due to uncertainties and problems occurring in real time the system dynamic modeling may not be suitable [13-15]

In recent years, many researchers are also using agent based distributed modeling of the supply chain $[5,16,17]$. One or several agents can be used to represent each entity in the SCN. Each agent is assigned with a local objective and global objective. With the advent of mobile agents which can run on lighter platforms, the use of agent to collect the information and to aid in the decision has become popular. Moreover, the agent paradigm is a natural metaphor for network organizations, since companies prefer maximizing their own profit than the profit of the supply chain [16]. Multi agent based modeling offers a way to elaborate the supply chain as the agents are autonomous and the agent rules can be defined in advance. The distributed decision nature of the multi agent systems makes it easier to add other entities in the local environment. Entities leaving the SCN in the middle will not affect the entire SCN to great extent. The agent rule frame work provides certain amount of trust among the partners as it eliminates the mistrust and deception among entities.

The major disadvantages with the multi agent based SCN modeling is:

- As there is no global view of the system theoretical optimisation, the optimization of the SCN can't be visualized.

- As per the system dynamic theory any system can be unstable [11], this theorem proves multi agent system, which is a system with multi autonomous systems, again can be unstable.

Again multi agent system also relay on the assumption that all the participating entities in the SCN are truly integrated and collaborating. However "true integration and collaboration" is highly difficult $[3,9,12,18-20]$. By considering these issues, in this work we considered the complex nonlinear modeling of the three echelon supply chain. 


\subsection{Nonlinear modeling of SCN}

A three echelon model is envisaged as shown in Figure 1 to describe a simple scenario in a very complex supply chain. The nonlinear supply chain models in the literature [10, 21], focus mainly on the specific task again becoming transaction oriented approach.

$$
\begin{array}{ll}
i & \text { Time period } \\
m & \text { Ratio of customer demand satisfied } \\
k & \text { Safety stock coefficient } \\
x_{i} & \begin{array}{l}
\text { The quantity demanded for products in current } \\
\text { period }
\end{array} \\
y_{i} & \begin{array}{l}
\text { The quantity distributors can supply in current } \\
\text { period }
\end{array} \\
z_{i} & \begin{array}{l}
\text { The quantity produced in current period depend on } \\
\text { the order }
\end{array}
\end{array}
$$

Here in this work, we mainly focus on building a nonlinear supply chain model that can exhibit more complexity and near to the realistic scenario. Figure 1 also illustrates the relationship between the three layers. The orders they make may not be equal to orders they receive. The order out quantity depends not only on how much inventory you have already but also how much you want to supply out. Order out quantity at retailer depends on the ratio $m$ at which the demand is satisfied during the previous order. Distributor needs to take into consideration among other things, the distortion rate $r$ that can occur in the inventories. The producer needs to take care about the safety stock $k$ in order to avoid the small production batches. These phenomena are explained below with mathematical modeling.

We consider that the demand information is transmitted within the layers of the supply chain with a delay of one unit time. As illustrated in Fig. 1, the ordering quantity is not same as the requested order quantity at any level. The order quantity at current period of time at retailer is linearly coupled with the distributor and it is influenced by how much of demand is satisfied in previous period of time. This phenomenon can be represented as shown in Eq.1.

$$
x_{i}=m\left(y_{i-1}-x_{i-1}\right)
$$

Here $m$ is the ratio at which the demand is satisfied. As it appears from Fig. 1, the dependency or coupling of distributor on producer and retailer is no more linear. Indeed the distributor needs to take the combined effect of retailer and producer into consideration before making his order, i.e., quadratic coupling. Apart from this, the distributor also needs to take into consideration the expected loss rate or distortion that can take place on the producer's supplies. This can be expressed mathematically as shown in Eq. 2.

$$
y_{i}=x_{i-1}\left(r-z_{i-1}\right)
$$

Where $r$ is the distortion coefficient. The production quantity from the producer unit typically depends on the distributor's orders and the safety stock. However the distributors' orders again depend on the retailer's orders, i.e., the producer needs to take the combined effect of retailer and distributor into account before making production decisions. This can be represented as

$$
z_{i}=x_{i-1} y_{i-1}+k z_{i-1}
$$

Eqs. 1-3 represent the quantity demanded by customers (Eq. 1), the inventory level of distributors (Eq. 2) and the quantity produced by producers (Eq. 3).

- Where $x_{i}<0$ denotes that the supply is less than customers demand in the previous period

- $\quad \mathrm{y}_{\mathrm{i}}<0$ denotes that information is severely distorted and no adjustment is necessary to inventory level

- $\quad \mathrm{z}_{\mathrm{i}}<0$ denotes the cases of overstock or return and hence no new productions.

Evidently the continuous forms of Eqs. 1-3 can be rewritten as Lorenz equations of state:

$$
\begin{aligned}
& \dot{x}=\sigma(y-x) \\
& \dot{y}=r x-y-x z \\
& \dot{z}=x y-b z
\end{aligned}
$$

Depending upon the parameter values, this model produces a wide variety of nonlinear features. Similar model is also proposed by the authors in [9]. In Figure 2, we showed the reference model with the parameter values $\sigma=15, r=29$ and $b=2 / 3$ to illustrate the regular state of the reference supply chain model.

In this work, we consider both internal and external perturbation

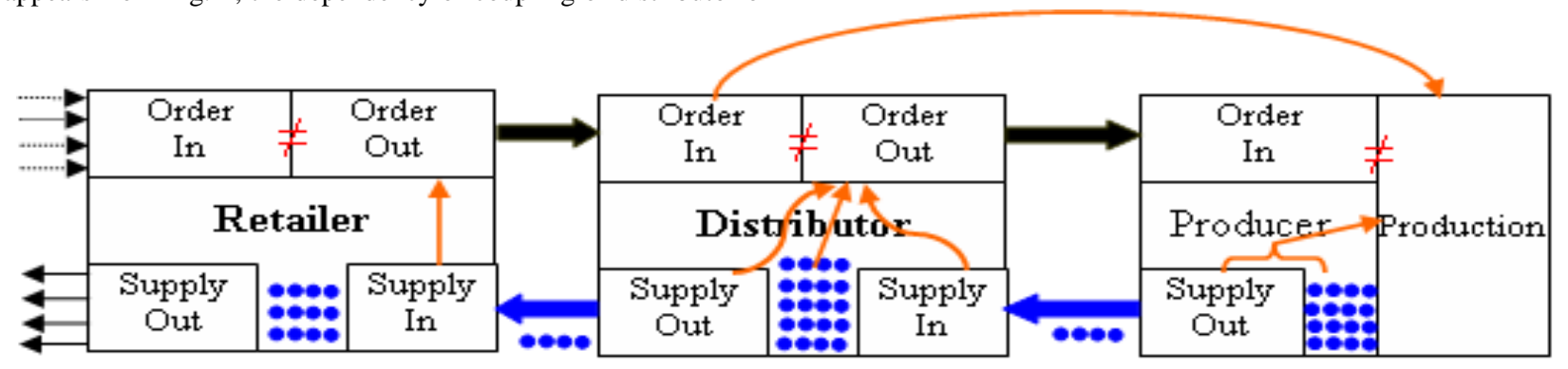

Figure 1: Three level supply chain mode1 
of the supply chain network. The internal perturbation, which is linear, is in large part due to the internal structure of the system. We consider the external perturbation as a nonlinear perturbation which is in general from the out side system. Assume the equations of state of Eqs. 4-6 in perturbation as follows by considering both internal perturbation and external perturbation

$$
\begin{aligned}
& \dot{x}^{\prime}=(\sigma+d \sigma)\left(y^{\prime}-x^{\prime}\right)+d_{1} \\
& \dot{y}^{\prime}=(r+d r) x^{\prime}-y^{\prime}-x^{\prime} z^{\prime}+d_{2} \\
& \dot{z}^{\prime}=x^{\prime} y^{\prime}-(b+d b) z^{\prime}+d_{3}
\end{aligned}
$$

Where $d \sigma, d r$ and $d b$ represents the internal perturbations and $d_{i}(i=1,2,3)$ represents the external perturbations.
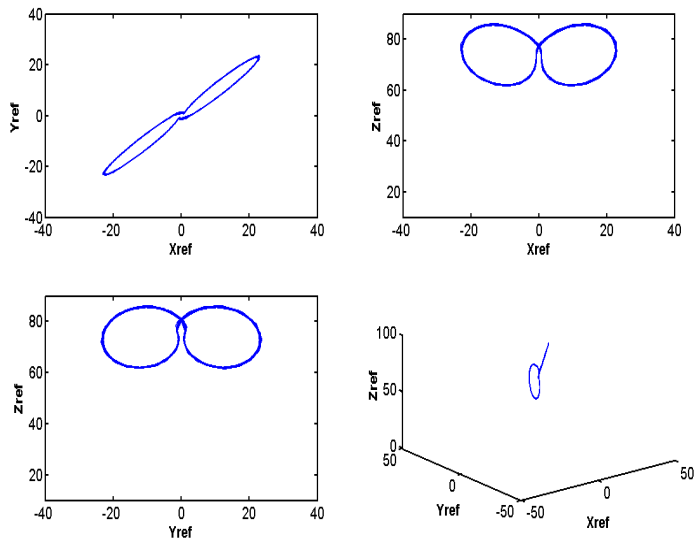

Figure 2: Reference SCN with given coefficients

\section{Enablers of self-organized SCN}

After establishing a viable complex nonlinear model, in this section we will discuss the key differences between traditional and self-organized supply chains in order to enlist the required key enablers of self-organized SCN. As we discussed in the section 1, information flow is the one of the key aspect to consider in any efficient supply chain. The information availability to the other entities in the SCN is one of the fundamental building blocks for any sort of dynamic decision making. As the competition between supply chains is gaining more importance than the competition between entities, information availability brings the visibility among the entities. Having information available, the focus moves on to the quality of information available. The visibility obtained by the availability of the information becomes nullified if the quality of the information is poor. In order to accomplish the selforganized supply chains, total visibility in terms of product order, monitoring of the order status by all the entities in the SCN is needed.

After overcoming the information visibility barrier, the next step towards the self-organization is the access of the information when ever it is required without any time delay. Information flow is sequential and very slow in the traditional supply chain. The information flow in case of self-organized SCN is parallel and dynamic. The timeliness of the information is very crucial where the response reaction time is very short due to the competition between SCNs. Many companies have looked to the modern web and wireless technologies to make the information available in a timeliness manner $[2,20]$. The integration efforts and the collaboration for the processes with in the SCN certainly improved communication by means of EDI and current internet based web information exchanges. Better information (point of sale data and the Collaborative Planning, Forecasting, and Replenishment, CPFR, initiatives), and a general willingness to work more closely together made the timeliness of information possible to certain extent. But the efficiencies have been gained through improvements that any executive can effect at his or her own workplace by putting in place the appropriate company-wide initiatives aimed at improving the internal business process.

The other key enabler towards the self-organized SCN is the mobile agent technology. The dynamic and distributed nature of the SCN requires software that not only makes the information available in a timeliness manner, but also intelligently collects information and process the information to take decisions in distributed environment. The agent based methods complement the enterprise resource planning (ERP) systems [22]. The multi agent based systems are also used in stand alone to aid the decision making of the executives $[16,23]$.

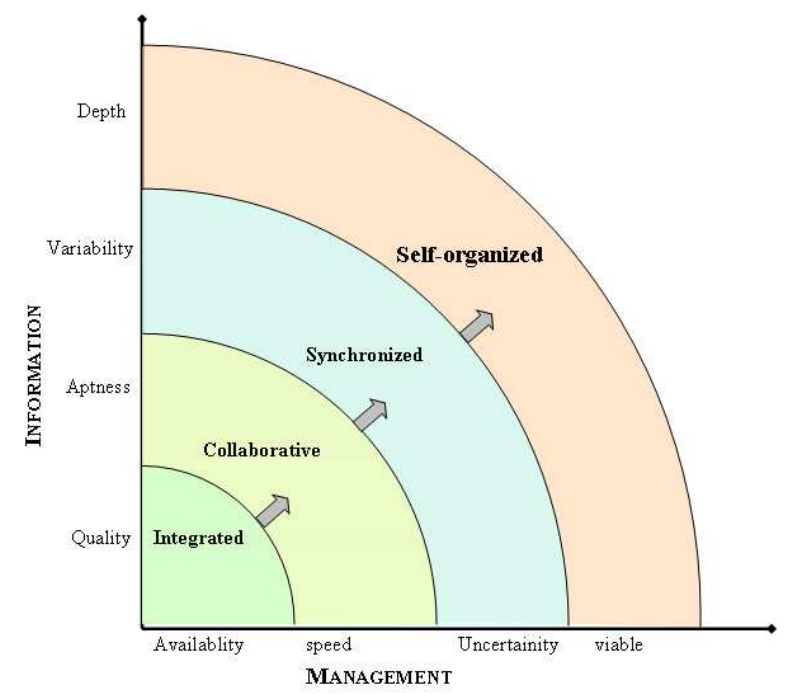

Figure 3: Key stages to the evolution of self-organized supply chain networks

To create the self-organized SCN, we also need the synchronization of the information that is available through various methods discussed in this section and in previous sections due to the fact that there is a certain amount of uncertainty exists. The uncertainties that exist in the SCN can force the $\mathrm{SCN}$ to go into chaotic state or saturation state as described in [2, 3, 8, 9, 24]. This makes the synchronization to be one of the key stages in the evolution of the self-organized supply chains (see figure 3 ). We have proposed adaptive numerical codes (algorithms and/or tools) for the systematic and automatic control of the internal thresholds in the supply chains to bring the SCN again to its stable state. However due to computational complexity of controlling the 
nonlinear system with adaptive numerical codes, in this work we propose an adaptive synchronization with the help of analog simulation based on CNN (Cellular Neural Network) technology.

\section{Analog simulation based approach}

The causes of supply chain instability can be broadly classified into two categories. The first cause is the dynamical and nonlinear character of the motions (i.e. material/products flow, information exchanges, etc...) between different entities in supply chains. The second cause originates from the effects of both external and internal perturbations to which the supply chain is subjected. An optimal management of the information flows within the supply chains may be of high importance in order to alleviate the effects resulting in negative consequences on the flows within the supply chains. In this work, we are considering the effect of external perturbation on the SCN. We considered the same reference model of the three echelon supply (see Figure 2) and show that a particular change of the key parameters of the external perturbations can lead to well- known bullwhip and chaotic phenomena. This is clearly illustrated in Figure 4 which shows chaotic attractors representing the perturbed state of the supply chain. In order to synchronize the system adaptively, we are using the analog simulation method based on CNN because the analog simulation is much faster due to the fact that a $\mathrm{CNN}$ is a programmable circuit.
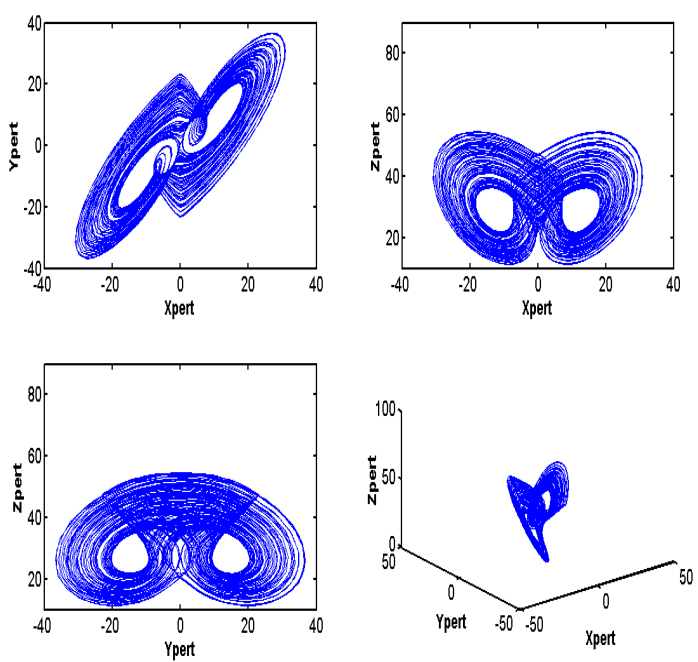

Figure 4: Chaotic state of the supply chain due to external perturbations

Cellular Neural Networks were introduced by Leon O. Chua and Yang from university of California at Berkeley in 1988. This type of neural networks is a somehow reduced version of Hopfield Neural Network. The idea of Chua was to use an array of essentially simple coupled nonlinear dynamic circuits, called cells, to process large amounts of information in real time. This concept was inspired by the Cellular Automata and the Neural Network architectures. The basic cell proposed by Chua is of the form shown in Figure 5. The basic building block for the CNN is the isolated cell.

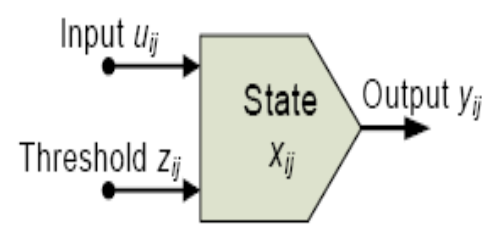

Figure5: Isolated CNN cell

This cell is a lumped circuit containing both linear and nonlinear elements as shown in Figure 6 together with the output function

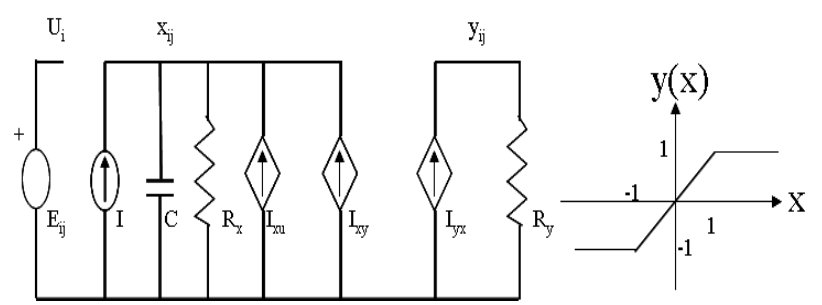

Figure 6: Isolated cell circuit representation with out put function

The model proposed by Chua is indeed a nonlinear circuit; however some authors refer it as linear CNN to emphasize the linearity of the Voltage Controlled Current Source determining the coupling. In [25], authors proved that it is indeed nonlinear circuit and proposed the state equation describing the CNN dynamics as expressed in Eq. 10.

$$
\dot{x}_{i}=-x_{i}+\sum_{i, j}\left(\hat{A}_{i, j} * x_{j}+A_{i, j} * y_{j}+B_{i, j} * u_{j}\right)+I_{i}
$$

Where the coupling coefficients $\hat{A}, A$ and $B$ are the self feedback template, feedback template and control templates respectively. CNNs are particularly interesting because of the programmable nature i.e. changeable templates.

In terms of the mathematical structure the considered model is much more complicated than the Chua's equation because it requires two nonlinear functions of two variables. Interestingly Brown [26] presented the generalization for the Chua's equations to obtain the Lorenz oscillations. In this work we consider the generalization proposed by Brown to simplify the complexity of the Lorenz model without loosing the nonlinearity presented in the model. The system model described in the Eq. 4-6 can be re arranged into Eq. 11 in the generalized form described by Brown [26].

$$
\left[\begin{array}{ccc}
-\sigma & \sigma & 0 \\
\left(r-z_{0}\right) & -1 & -x_{0} g(x) \\
x_{0} g(x) & 0 & -b
\end{array}\right] *\left[\begin{array}{c}
x-x_{0} g(x) \\
y-x_{0} g(x) \\
z-z_{0}
\end{array}\right]
$$

The template values for the generalized equation in comparison with the CNN state equation shown in Eq. 10 are as follows 


$$
\begin{array}{lll}
\hat{A}_{11}=-\sigma+1 & \hat{A}_{21}=r-z_{0} & \hat{A}_{31}=x_{0} * g(x) \\
\hat{A}_{12}=\sigma & \hat{A}_{22}=0 & \hat{A}_{32}=0 \\
\hat{A}_{12}=0 & \hat{A}_{23}=-x_{0} * g(x) & \hat{A}_{33}=-b+1 \\
& A_{21}=-r * x_{0}+2 * x_{0} * z_{0} & A_{31}=x_{0}^{2} * g(x) \\
& A_{22}=y_{0} & I_{3}=b * z_{0}
\end{array}
$$

This realization of the Lorenz model from the generalized Chua's model helps in developing the analog simulation environment over the Matlab/Simulink ${ }^{\circledR}$. We develop a method based on an adaptive algorithm for the automatic cancellation of the effects of the external perturbations by re-adjusting the internal thresholds, the internal thresholds are effectively represented in the templates. The template values are adaptively changed till it gives the stable system. The external perturbation is represented with the threshold coefficient $I$. The synchronized system obtained by varying the internal threshold, template constants, is shown in Figure 7, which almost resembles the reference system in Figure 1.
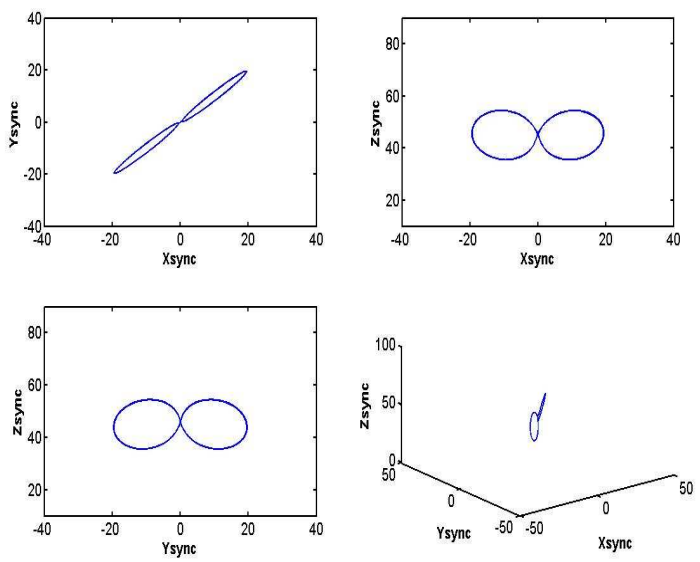

Figure 7: Alleviation of the chaotic effect caused by external perturbation with adaptive numerical code

\section{Conclusions}

Self-organization of supply chain is a complex issue involving many key stages. Even though we have the technology to share the information in near real time, SCN comes with various uncertain issues. The competition between supply networks is only a global view. There exists competition between local entities with in the supply chain. Even though willingness for the integration and the agreements for the cooperation are the key stages, the synchronization plays an important role due to the aspects discussed throughout this paper. This paper considers only external perturbation creating chaotic behavior, how ever there exists also internal perturbation within one entity affecting the performance of the entire supply chain. The complexity level increases when there in multiple internal perturbations at different entities coupled with external perturbation. The future work will focus in this direction. It would also be of great interest to analyze the coupling between different structures of supply chains and the achievement of synchronization within them. It is well known that in practice, many commercial supply chains are coupled.

\section{References}

[1] J. B. Ayers, Handbook of Supply Chain Management, 2nd ed. New York: Auer Bach Publications, April 2006.

[2] K. R. Anne, J. C. Chedjou, and K.Kyamakya, "Modeling of a three echelon supply chain: Stability analysis and synchronization issues," in International workshop on nonlinear dynamics and synchronization, Klagenfurt, Austria, 2008.

[3] H. B. Hwarng and N. Xie, "Understanding supply chain dynamics: A chaos perspective," European journal of operations research, pp. 1163-1178, 2006.

[4] T. Skjoett-Larsen, C. Thernøe, and C. Andresen, "Supply chain collaboration: Theoretical perspectives and empirical evidence " International Journal of Physical Distribution \& Logistics Management, vol. 33, pp. 531 - 549, 2003.

[5] B. D. Jespersen and T. Skjott-Larsen, Supply Chain Management: In Theory and Practice: Copenhagen Business School Press DK, 2005.

[6] S. E. Fawcett and G. M. Magnan, "The rhetoric and reality of supply chain integration," International Journal of Physical Distribution \& Logistics Management, vol. 32, pp. 339-361, April 2002.

[7] M. Holweg, S. Disney, J. Holmström, and J. Smårosa, "Supply Chain Collaboration: Making Sense of the Strategy Continuum," European Management Journal, vol. 23, pp. 170-181 April 2005.

[8] K. R. Anne, J. C. Chedjou, and K. Kyamakya, "Bifurcation analysis and synchronization issues in a three echelon supply chain network," in Logistics Research Network annual conference Liverpool, UK, 2008.

[9] Z. Lei, L. Yi-jun, and X. Yao-qun, "Chaos Synchronization of Bullwhip Effect in a Supply Chain," in Management Science and Engineering, 2006. ICMSE '06. 2006 International Conference on, Lille, Date: 5-7 Oct. 2006, pp. 557-560.

[10] C. C. Poirier, Using Models to Improve the Supply Chain Routledge, USA August 2003.

[11] J. W. Forrester, Industrial Dynamics. Portland: Productivity press, 1961.

[12] J. D. Sterman, "Modeling managerial behavior: misperceptions of feedback in a dynamic decision making experiment," Management Science vol. 35 pp. 321 - 339 1989

[13] B. J. Angerhofer and M. C. Angelides, "System dyanamics modeling of supply chain management: Research review," in Winter Simulation Conference, 2000.

[14] H. Laurikkala, H. Vilkman, M. Ek, H. Koivisto, and G.-Y. Xiong, "Modeling and control of supply chain with system theory," Tampere University of Technology, Institute of Machine Design 2003.

[15] F. Chen, Z. Drezner, J. K. Ryan, and D. Simchi-Levi, "Quantifying the Bullwhip Effect in a Simple Supply Chain: The Impact of Forecasting, Lead Times, and Information," Management Science vol. 46, pp. 436 - 4432000. 
[16] M. E. Nissen, "Agent-Based Supply Chain Integration," Information Technology and Management, vol. 2, pp. 289 312, 2001.

[17] H. Stadtler and C. Kilger, Supply Chain Management and Advanced Planning: Concepts, Models, Software, and Case Studies: Springer, 2000.

[18] J. Dejonckheere, S. M. Disney, M. R. Lambrecht, and D. R. Towillb, "Measuring and avoiding the bullwhip effect: A control theoretic approach," European Journal of Operational Research vol. 147, pp. 567-590, 16June 2003 June 2003.

[19] A. Akintoye, "A survey of supply chain collaboration and management in the UK construction industry," European Journal of Purchasing \& Supply Management, vol. 6, pp. 159-168 December 2000.

[20] M. Khouja, "Synchronization in supply chains: implications for design and management," journal of the operations research society, pp. 984-994, 2003.
[21] L. d. Giacomo and G. Patrizi, "Dynamic nonlinear modelization of operational supply chain," Journal of Global Optimization, vol. 34, pp. 503-534, 2006.

[22] SAP, "Adaptive supply chain networks," 2002.

[23] M. T. Frohlich and R. Westbrook, "Arcs of integration: an international study of supply chain strategies," Journal of Operations Management, vol. 19, pp. 185 - 200, February 2001.

[24] R. Wilding, "The supply chain complexity triangle: Uncertainty generation in the supply chain " International Journal of Physical Distribution \& Logistics Management vol. 28, pp. 599 - 6161998.

[25] G. Manganaro, P. Arena, and L. Fortuna, Cellular Neural Networks: Chaos, Complexity and VLSI Processing. Berlin: Springer.

[26] R. Brown, "Generalizations of the Chua Equations," International Journal of Bifurcation \& Chaos in Applied Sciences \& Engineering, vol. 2, pp. 889 - 909, 1992. 